\title{
NUMERICAL RECKONING FIXED POINTS FOR BERINDE MAPPINGS VIA A FASTER ITERATION PROCESS
}

\author{
Osman Alagoz, Birol Gunduz and Sezgin Akbulut
}

\begin{abstract}
In this paper we prove that the $M$-iteration process converges strongly faster than $S$-iteration and Picard- $S$ iteration processes. Moreover, the $M-$ iteration process is faster than the $S_{n}$ iteration process with a sufficient condition for weak contractive mapping defined on a normed linear space. We also give two numerical reckoning examples to support our main theorem. For approximating fixed points, all codes were written in MAPLE (C) 2018 All rights reserved.
\end{abstract}

Keywords: Iteration process, fixed point, weak contractive mapping, normed linear space.

\section{Introduction and Preliminaries}

Let $K$ be a non-empty convex subset of a Banach space $X$ and let $T: K \rightarrow K$ be a mapping. A point $p$ is called the fixed point of a mapping $t$ if $T p=p$ and $F(T)$ represents the set of all fixed points of the mapping $T$.

It is well known that any linear or non-linear equation including differential equations and integral equations, can be transferred into a fixed point problem. For example, non-linear equations $x^{2}-\sin x=0$, and $x^{3} \ln x+e^{x}=0$ cannot be solved easily. But, after transferring them into fixed point problems such as $T x=x$, we can approximate the fixed point or the fixed points of the mapping of $T$ with the help of iteration schemes. Thus, it is clear that any fixed point of $T$ is also a solution of the corresponding equations.

One of the main conclusions which guarantees the existence of a fixed point was given by S. Banach in 1922 which is also called the Banach contraction principle and given as follows:

Theorem 1.1. Let $(X, d)$ be a complete metric space and $T: X \rightarrow X$ be a mapping. If there exists a $k \in[0,1)$ such that

$$
d(T x, T y) \leq k d(x, y)
$$

Received March 19, 2018; accepted April 12, 2018

2010 Mathematics Subject Classification. Primary 47H09; Secondary 47H10 
for all $x, y \in X$, then $T$ has a unique fixed point.

Then since the Banach contraction principle was defined, many researchers have studied fixed point theory on different classes of mapping, on different types of spaces, and on different iteration processes.

In this paper, we give some useful results about some iteration schemes for finding fixed points of $T$. Firstly, we give some well-known iteration processes. Let $(X, d)$ be a metric space and let $T: X \rightarrow X$ be a mapping and let $\left\{\alpha_{n}\right\},\left\{\beta_{n}\right\}$ and $\left\{\gamma_{n}\right\}$ be real sequences in $(0,1]$. For $x_{0} \in X$,

- Picard iteration (1890) [12]

$$
x_{n+1}=T x_{n}, \quad n=0,1,2, \ldots
$$

- Mann iteration (1953) [9]

$$
x_{n+1}=\left(1-\alpha_{n}\right) x_{n}+\alpha_{n} T x_{n}, \quad n=0,1,2, \ldots
$$

- Ishikawa iteration (1974) [7]

$$
\begin{cases}x_{n+1} & =\left(1-\alpha_{n}\right) x_{n}+\alpha_{n} T y_{n} \\ y_{n} & =\left(1-\beta_{n}\right) x_{n}+\beta_{n} T x_{n}, \quad n=0,1,2, \ldots\end{cases}
$$

- S-iteration (2007) [2]

$$
\begin{cases}\xi_{n+1} & =\left(1-\alpha_{n}\right) T \xi_{n}+\alpha_{n} T \mu_{n} \\ \mu_{n} & =\left(1-\beta_{n}\right) \xi_{n}+\beta_{n} T \xi_{n}, \quad n=0,1,2, \ldots\end{cases}
$$

- Picard-S iteration (2014) [6]

$$
\begin{cases}p_{n+1} & =T q_{n} \\ q_{n} & =\left(1-\alpha_{n}\right) T p_{n}+\alpha_{n} T r_{n} \\ r_{n} & =\left(1-\beta_{n}\right) p_{n}+\beta_{n} T p_{n}\end{cases}
$$

- $S_{n}$ iteration (2016) [14]

$$
\left\{\begin{array}{l}
u_{n+1}=\left(1-\alpha_{n}\right) T w_{n}+\alpha_{n} T v_{n} \\
v_{n}=\left(1-\beta_{n}\right) u_{n}+\beta_{n} v_{n} \\
w_{n}=\left(1-\gamma_{n}\right) u_{n}+\gamma_{n} T u_{n}, \quad n=0,1,2, \ldots
\end{array}\right.
$$

- $M$-iteration (2018) [16]

$$
\begin{cases}x_{n+1} & =T y_{n} \\ y_{n} & =T z_{n} \\ z_{n} & =\left(1-\alpha_{n}\right) x_{n}+\alpha_{n} T x_{n}, \quad n=0,1,2, \ldots\end{cases}
$$


In 2004, Berinde [4] introduced the concept of contractive mappings on metric space $(X, d)$ as follows.

Definition 1.1. Let $T$ be a mapping on a metric space $(X, d)$. Then $T$ is called a Berinde mapping if there exists $\delta \in[0,1)$ and $L \in[0, \infty)$ such that

$$
d(T x, T y) \leq \delta d(x, y)+L d(x, T x) \quad \forall x, y \in X
$$

for all $x, y \in X$.

He also proved that any Zamfirescu mapping satisfies the weak contractive condition. Thus, the class of weak contractive mappings is wider than the class of Zamfirescu mapping. We refer the readers to $[17,3]$ to learn more about Zamfirescu and Berinde mapping.

In order to compare convergence rates between two iteration processes, we use the following useful definitions.

Definition 1.2. [4] Let $\left\{x_{n}\right\}$ and $\left\{y_{n}\right\}$ be two sequences of real numbers that converge to $x$ and $y$, respectively and suppose that there exists

$$
L: \lim _{n \rightarrow \infty} \frac{\left|x_{n}-x\right|}{\left|y_{n}-y\right|}
$$

1. If $L=0$, then $\left\{x_{n}\right\}$ converges faster to $x$ than $\left\{y_{n}\right\}$ to $y$.

2. If $0<L<\infty$, then $\left\{x_{n}\right\}$ and $\left\{y_{n}\right\}$ have the same rate of convergence.

Definition 1.3. [1] Let $(X,\|\cdot\|)$ be a normed linear space and $\left\{u_{n}\right\}$ and $\left\{v_{n}\right\}$ converging to the same point $p \in X$ and following the error estimates

$$
\begin{aligned}
&\left\|u_{n}-p\right\| \leq a_{n} \quad \forall n \in \mathbb{N} ; \\
&\left\|v_{n}-p\right\| \leq b_{n} \quad \forall n \in \mathbb{N} ;
\end{aligned}
$$

are available, where $\left\{a_{n}\right\}$ and $\left\{b_{n}\right\}$ are two sequences of positive numbers. If $\left\{a_{n}\right\}$ converges faster than $\left\{b_{n}\right\}$ then $\left\{u_{n}\right\}$ converges faster than $\left\{v_{n}\right\}$ to $p$.

\section{Approximation Results}

Recently, Gursoy and Karakaya [6] proved that the Picard- $S$ iteration process converges faster than all Picard [12], Mann [9], Ishikawa [7], Noor [10], SP [11], CR [5], S [2], S* [8], Abbas [1], Normal-S [13] and two-step Mann [15] iteration processes for contraction mappings.

In 2016, Sintunavarat and Pitea [14] defined a new three step iteration which is called $S_{n}$ iteration. They also showed that their iteration converges faster than Mann, Ishikawa and $S$-iteration processes for mappings satisfying Berinde contractive condition. 
In 2018, Ullah and Arshad [16] defined a new three step iteration process, called $M$-iteration process for finding fixed points of mappings and they get some convergence results for Suzuki generalized nonexpansive mappings in uniformly convex Banach spaces. They also showed that the $M$-iteration process converges faster than the Picard- $S$ iteration and the $S$-iteration process for Suzuki generalized nonexpansive mappings.

Our purpose in this paper is to prove that the M-iteration process converges faster than the $S_{n}$ iteration process with a sufficient condition and faster than the $S$-iteration and Picard- $S$ iteration processes for weak contractive mappings. We support our result with two numerical examples.

Theorem 2.1. Let $K$ be a non-empty closed convex subset of a Banach space $(X,\|\cdot\|)$ and $T: K \rightarrow K$ be a mapping satisfying the weak contractive condition (1.8) with a fixed point $p$. Suppose that the sequences $\left\{x_{n}\right\},\left\{u_{n}\right\},\left\{p_{n}\right\}$ and $\left\{\xi_{n}\right\}$ are defined by the iteration processes $M, S_{n}$, Picard-S and S-iteration processes, respectively. Also the sequences $\left\{\alpha_{n}\right\},\left\{\beta_{n}\right\}$ and $\left\{\gamma_{n}\right\}$ are in $[\alpha, 1-\alpha],[\beta, 1-\beta]$, and $[\gamma, 1-\gamma]$, respectively, $\alpha, \beta, \gamma \in\left(0, \frac{1}{2}\right)$. Then the $M$-iteration process converges faster than the $S$ and Picard-S iteration processes. Moreover, if $\alpha(2-\gamma)<\gamma$ then the $M$-iteration process is also faster than the $S_{n}$-iteration process.

Proof. By using the $M$-iteration, we can get the following result

$$
\begin{aligned}
\left\|x_{n+1}-p\right\| & =\left\|T y_{n}-p\right\| \\
& \leq \delta\left\|y_{n}-p\right\| \\
& =\delta\left\|T z_{n}-p\right\| \\
& \leq \delta^{2}\left\|z_{n}-p\right\| \\
& =\delta^{2}\left\|\left(1-\alpha_{n}\right) x_{n}+\alpha_{n} T x_{n}-p\right\| \\
& \leq \delta^{2}\left[\left(1-\alpha_{n}\right)\left\|x_{n}-p\right\|+\alpha_{n} \delta\left\|x_{n}-p\right\|\right] \\
& =\left(1-(1-\delta) \alpha_{n}\right) \delta^{2}\left\|x_{n}-p\right\|
\end{aligned}
$$

for all $n \in \mathbb{N}$. Therefore,

$$
\left\|x_{n}-p\right\| \leq\left\{(1-(1-\delta) \alpha) \delta^{2}\right\}^{n}\left\|x_{0}-p\right\|
$$

for all $n \in \mathbb{N}$. Choose

$$
a_{n}:=\left\{(1-(1-\delta) \alpha) \delta^{2}\right\}^{n}\left\|x_{0}-p\right\| .
$$

By using the Picard- $S$ iteration, we get

$$
\begin{aligned}
\left\|r_{n}-p\right\| & \leq\left\|\left(1-\beta_{n}\right) p_{n}+\beta_{n} T p_{n}-p\right\| \\
& \leq\left(1-\beta_{n}\right)\left\|p_{n}-p\right\|+\beta_{n}\left\|T p_{n}-p\right\| \\
& \leq\left(1-\beta_{n}\right)\left\|p_{n}-p\right\|+\beta_{n} \delta\left\|p_{n}-p\right\| \\
& =\left(1-(1-\delta) \beta_{n}\right)\left\|p_{n}-p\right\| .
\end{aligned}
$$


Using the Picard-S again and from (2.2), we have

$$
\begin{aligned}
\left\|q_{n}-p\right\| & \leq\left\|\left(1-\alpha_{n}\right) T p_{n}+\alpha_{n} T r_{n}-p\right\| \\
& \leq\left(1-\alpha_{n}\right)\left\|T p_{n}-p\right\|+\alpha_{n}\left\|T r_{n}-p\right\| \\
& \leq\left(1-\alpha_{n}\right) \delta\left\|p_{n}-p\right\|+\alpha_{n} \delta\left\|r_{n}-p\right\| \\
& \leq\left(1-\alpha_{n}\right) \delta\left\|p_{n}-p\right\|+\alpha_{n} \delta\left(1-(1-\delta) \beta_{n}\right)\left\|p_{n}-p\right\| \\
& =\left(1-(1-\delta) \alpha_{n} \beta_{n}\right) \delta .\left\|p_{n}-p\right\| .
\end{aligned}
$$

From (2.3), we get

$$
\begin{aligned}
\left\|p_{n+1}-p\right\| & =\left\|T q_{n}-p\right\| \\
& \leq \delta\left\|q_{n}-p\right\| \\
& \leq\left(1-(1-\delta) \alpha_{n} \beta_{n}\right) \delta^{2}\left\|p_{n}-p\right\| .
\end{aligned}
$$

for all $n \in \mathbb{N}$. Thus,

$$
\left\|p_{n}-p\right\| \leq\left\{(1-(1-\delta) \alpha \beta) \delta^{2}\right\}^{n}\left\|p_{0}-p\right\|
$$

for all $n \in \mathbb{N}$. Let

$$
b_{n}:=\left\{(1-(1-\delta) \alpha \beta) \delta^{2}\right\}^{n}\left\|p_{0}-p\right\| .
$$

As proved in Theorem 2.1 of [14], we have

$$
\left\|u_{n}-p\right\| \leq\left\{1-(1-\delta) \beta\left[\gamma_{n}-\alpha_{n}+\alpha_{n} \gamma_{n}\right]\right\}^{n}\left\|u_{0}-p\right\|
$$

and

$$
\left\|\xi_{n}-p\right\| \leq[1-(1-\delta) \alpha \beta]^{n}\left\|\xi_{0}-p\right\|
$$

for all $n \in \mathbb{N}$. Choose

$$
c_{n}:=\{1-(1-\delta) \beta[\gamma-\alpha+\alpha \cdot \gamma]\}^{n}\left\|u_{0}-p\right\| .
$$

and

$$
d_{n}:=[1-(1-\delta) \alpha \beta]^{n}\left\|\xi_{0}-p\right\|
$$

Since $\alpha(2-\gamma)<\gamma$, we obtain

$$
1-(1-\delta) \beta(\gamma-\alpha+\alpha \cdot \gamma)<1-(1-\delta) \alpha \beta<1
$$

Now using the definition (1.2) and the definition (1.3) we get the following results.

$$
\begin{aligned}
\lim _{n \rightarrow \infty}\left\|x_{n}-p\right\| & \leq \lim _{n \rightarrow \infty}\left[(1-(1-\delta) \alpha) \delta^{2}\right]^{n}\left\|x_{0}-p\right\|=0, \\
\lim _{n \rightarrow \infty}\left\|p_{n}-p\right\| \leq \lim _{n \rightarrow \infty}\left[(1-(1-\delta) \alpha \beta) \delta^{2}\right]^{n}\left\|p_{0}-p\right\|=0, & \leq \lim _{n \rightarrow \infty}[1-(1-\delta) \beta[\gamma-\alpha+\alpha \cdot \gamma]]^{n}\left\|u_{0}-p\right\|=0, \\
\lim _{n \rightarrow \infty}\left\|u_{n}-p\right\| \leq \lim _{n \rightarrow \infty}[1-(1-\delta) \alpha \beta]^{n}\left\|\xi_{0}-p\right\|=0 . &
\end{aligned}
$$

Now we give convergence rates of the above iterations as follows; 
- $\lim _{n \rightarrow \infty} \frac{a_{n}}{b_{n}}=\frac{(1-(1-\delta) \alpha)^{n} \delta^{2 n}}{(1-(1-\delta) \alpha \beta)^{n} \delta^{2 n}} \cdot \frac{\left\|x_{0}-p\right\|}{\left\|p_{0}-p\right\|}=0$

- $\lim _{n \rightarrow \infty} \frac{a_{n}}{c_{n}}=\frac{(1-(1-\delta) \alpha)^{n} \delta^{2 n}}{[1-(1-\delta) \beta[\gamma-\alpha+\alpha \cdot \gamma]]^{n}} \cdot \frac{\left\|x_{0}-p\right\|}{\left\|u_{0}-p\right\|}=0$,

- $\lim _{n \rightarrow \infty} \frac{a_{n}}{d_{n}}=\frac{(1-(1-\delta) \alpha)^{n} \delta^{2 n}}{[1-(1-\delta) \alpha \beta]^{n}} \cdot \frac{\left\|x_{0}-p\right\|}{\left\|\xi_{0}-p\right\|}=0$.

Therefore, the conclusion follows.

\section{Numerical Results}

Now we give numerical examples to support our theorem. In both examples, we choose functions satisfying the weak contraction condition. It can be understood easily with the help of the mean value theorem.

Example 3.1. Let $D=[-10,10]$ be a subset of a usual normed space $\mathbb{R}$ and let $T$ : $D \rightarrow D$ be a mapping such that $T x=\sin (\cos x)$ for all $x \in D$. Choose $\alpha=\beta=0.12$ and $\gamma=0.24$ and $\alpha_{n}=\beta_{n}=\gamma_{n}=0.25$ for all $n \in \mathbb{N}$. It is obvious that $T$ has a unique fixed point $p=0.69481969073079 \in D$. Moreover, the sequences of $\left\{\alpha_{n}\right\},\left\{\beta_{n}\right\}$ and $\left\{\gamma_{n}\right\}$ and the parameters $\alpha, \beta$ and $\gamma$ satisfy the condition of Theorem 2.1 .

For an arbitrary initial point $x_{0}=2$, the values of the iterations of $S$, Picard- $S$, $S_{n}$ and $M$ are given in Table 1 . Thus, it is obvious that the $M$-iteration process converges faster than all other iterations. Now we give the graphs of these iterations to show their convergence behaviours in Figure 1.

In the next example, we use an exponential function which also satisfies the weak contractive condition.

Example 3.2. Let $D=[0,5]$ be a subset of a usual normed space $\mathbb{R}$ and let $T: D \rightarrow D$ be a mapping such that $T x=e^{\frac{4}{4+x^{2}}}$ for all $x \in D$. Choose $\alpha=\beta=0.2, \gamma=0.45$ and $\alpha_{n}=\beta_{n}=\gamma_{n}=0.50$ for all $n \in \mathbb{N}$. It is obvious that $T$ has a unique fixed point $p=1.7579448713504 \in D$. Moreover, the sequences of $\left\{\alpha_{n}\right\},\left\{\beta_{n}\right\}$ and $\left\{\gamma_{n}\right\}$ and the parameters $\alpha, \beta$ and $\gamma$ satisfy the conditions of Theorem 2.1.

For an arbitrary initial point $x_{0}=5$, the values of the iterations of $S$, Picard- $S$, $S_{n}$ and $M$ are given in Table 2. Thus, it is obvious that the $M$-iteration process converges faster than all other iterations. Now we give the graphs of these iterations in Figure 2 to see their convergence behaviours. 


\begin{tabular}{|c|c|c|c|c|}
\hline Step & Siteration & Picard- $S$ & $S_{n}$ iteration & $M$ iteration \\
\hline 1 & 2.00000000000000 & 2.00000000000000 & 2.000000000000000 & 2.00000000000000 \\
\hline 2 & -0.2606345908683 & 0.82274676877756 & 0.059666857688088 & 0.83357849776340 \\
\hline 3 & 0.82742078111148 & 0.72063145818922 & 0.79298563965090 & 0.71374670426113 \\
\hline 4 & 0.63322481776167 & 0.69984773469901 & 0.66834020139151 & 0.69737415165409 \\
\hline 5 & 0.71929615165795 & 0.69579056568185 & 0.70061313646443 & 0.69516366956216 \\
\hline 6 & 0.68437145798799 & 0.69500682583460 & 0.69335657926482 & 0.69486599536890 \\
\hline 7 & 0.69915397516439 & 0.69485574834868 & 0.69515086974096 & 0.69482592374820 \\
\hline 8 & 0.69299964736283 & 0.69482663793094 & 0.69473790460681 & 0.69482052974562 \\
\hline 9 & 0.69558010394304 & 0.69482102922656 & 0.69483851648450 & 0.69481980366892 \\
\hline 10 & 0.69450131531509 & 0.69481994861403 & 0.69481510357798 & 0.69481970593317 \\
\hline 11 & 0.69495287230429 & 0.69481974041622 & 0.69482075802148 & 0.69481969277715 \\
\hline 12 & 0.69476395803525 & 0.69481970030350 & 0.69481943293436 & 0.69481969100624 \\
\hline 13 & 0.69484300965349 & 0.69481969257512 & 0.69481975113705 & 0.69481969076786 \\
\hline 14 & 0.69480993330735 & 0.69481969108613 & 0.69481967622364 & 0.69481969073577 \\
\hline 15 & 0.69482377345466 & 0.69481969079925 & 0.69481969414590 & 0.69481969073146 \\
\hline 16 & 0.69481798240850 & 0.69481969074398 & 0.69481968991371 & 0.69481969073088 \\
\hline 17 & 0.69482040553572 & 0.69481969073333 & 0.69481969092373 & 0.69481969073080 \\
\hline 18 & 0.69481939163787 & 0.69481969073127 & 0.69481969068473 & 0.69481969073079 \\
\hline 19 & 0.69481981587892 & 0.69481969073088 & 0.69481969074169 & 0.69481969073079 \\
\hline 20 & 0.69481963836559 & 0.69481969073080 & 0.69481969072819 & 0.69481969073079 \\
\hline 21 & 0.69481971264172 & 0.69481969073079 & 0.69481969073140 & 0.69481969073079 \\
\hline 22 & 0.69481968156270 & 0.69481969073079 & 0.69481969073065 & 0.69481969073079 \\
\hline 23 & 0.69481969456696 & 0.69481969073079 & 0.69481969073082 & 0.69481969073079 \\
\hline 24 & 0.69481968912563 & 0.69481969073079 & 0.69481969073078 & 0.69481969073079 \\
\hline 25 & 0.69481969140243 & 0.69481969073079 & 0.69481969073079 & 0.69481969073079 \\
\hline 26 & 0.69481969044976 & 0.69481969073079 & 0.69481969073079 & 0.69481969073079 \\
\hline 27 & 0.69481969084838 & 0.69481969073079 & 0.69481969073079 & 0.69481969073079 \\
\hline 28 & 0.69481969068158 & 0.69481969073079 & 0.69481969073079 & 0.69481969073079 \\
\hline 29 & 0.69481969075137 & 0.69481969073079 & 0.69481969073079 & 0.69481969073079 \\
\hline 30 & 0.69481969072217 & 0.69481969073079 & 0.69481969073079 & 0.69481969073079 \\
\hline$\cdot$ & & $\vdots$ & . & : \\
\hline 39 & 0.69481969073079 & 0.69481969073079 & 0.69481969073079 & 0.69481969073079 \\
\hline 40 & 0.69481969073079 & 0.69481969073079 & 0.69481969073079 & 0.69481969073079 \\
\hline
\end{tabular}

Table 3.1: Comparative results of Example 3.1 


\begin{tabular}{|c|c|c|c|c|}
\hline Step & $S$ iteration & Picard- $S$ & $S_{n}$ iteration & $M$ iteration \\
\hline 1 & 5.00000000000000 & 5.00000000000000 & 5.00000000000000 & 5.00000000000000 \\
\hline 2 & 1.42941764430671 & 1.93846644431469 & 1.40485245885178 & 1.87003729972110 \\
\hline 3 & 1.84419392945226 & 1.77947624628915 & 1.75019355009770 & 1.76199242097177 \\
\hline 4 & 1.73187212214594 & 1.76120146917096 & 1.75603091341028 & 1.75819390853271 \\
\hline 5 & 1.76700050742008 & 1.75849723073996 & 1.75778491609830 & 1.75796491928449 \\
\hline 6 & 1.75460689343929 & 1.75804523322895 & 1.75790258123863 & 1.75794676700153 \\
\hline 7 & 1.75923776754111 & 1.75796396996576 & 1.75794020608824 & 1.75794507130585 \\
\hline 8 & 1.75742781464024 & 1.75794862884936 & 1.75794356116350 & 1.75794489420287 \\
\hline 9 & 1.75815703047109 & 1.75794562943728 & 1.75794471266250 & 1.75794487412885 \\
\hline 10 & 1.75785610751453 & 1.75794502733560 & 1.75794482305018 & 1.75794487170534 \\
\hline 11 & 1.75798260400559 & 1.75794490395775 & 1.75794486551196 & 1.75794487139762 \\
\hline 12 & 1.75792862247490 & 1.75794487825579 & 1.75794486935670 & 1.75794487135688 \\
\hline 13 & 1.75795194515923 & 1.75794487282874 & 1.75794487112933 & 1.75794487135130 \\
\hline 14 & 1.75794176333736 & 1.75794487166981 & 1.75794487126058 & 1.75794487135052 \\
\hline 15 & 1.75794624777708 & 1.75794487141994 & 1.75794487134225 & 1.75794487135040 \\
\hline 16 & 1.75794425757424 & 1.75794487136563 & 1.7579448 & 1.757 \\
\hline 17 & 1.75794514670100 & 1.75794487135375 & 1.75794487135012 & 1.75794487135038 \\
\hline 18 & 1.75794474716322 & 1.75794487135112 & 1.75794487135015 & 1.75794487135038 \\
\hline 19 & 1.75794492762700 & 1.75794487135055 & 1.75794487135039 & 1.75794487135038 \\
\hline 20 & 1.75794484573944 & 1.75794487135042 & 1.75794487135036 & 1.75794487135038 \\
\hline 21 & 1.75794488305041 & 1.75794487135039 & 1.75794487135039 & 1.75794487135038 \\
\hline 22 & 1.7579448 & 1.75794 & 7135038 & 1.75794487135038 \\
\hline 23 & 1.75794 & 1.75794 & 1.757 & 1.757944 \\
\hline 24 & 1.75794487021279 & 1.75794487135038 & 1.75794487135038 & 1.75794487135038 \\
\hline 25 & 1.75794487187643 & 1.75794487135038 & 1.75794487135038 & 1.75794487135038 \\
\hline 26 & 1.75794487110653 & 1.75794487135038 & 1.75794487135038 & 1.75794487135038 \\
\hline 27 & 1.75794487146368 & 1.75794487135038 & 1.75794487135038 & 1.75794487135038 \\
\hline 28 & 1.75794487129764 & 1.75794487135038 & 1.75794487135038 & 1.75794487135038 \\
\hline 29 & 1.75794487137498 & 1.75794487135038 & 1.75794487135038 & 1.75794487135038 \\
\hline 30 & 1.75794487133888 & 1.75794487135038 & 1.75794487135038 & 1.75794487135038 \\
\hline$\vdots$ & $\vdots$ & : & : & : \\
\hline 39 & 1.75794487135039 & 1.75794487135038 & 1.75794487135038 & 1.75794487135038 \\
\hline 40 & 1.75794487135038 & 1.75794487135038 & 1.75794487135038 & 1.75794487135038 \\
\hline
\end{tabular}

Table 3.2: Comparative results of Example 3.2 


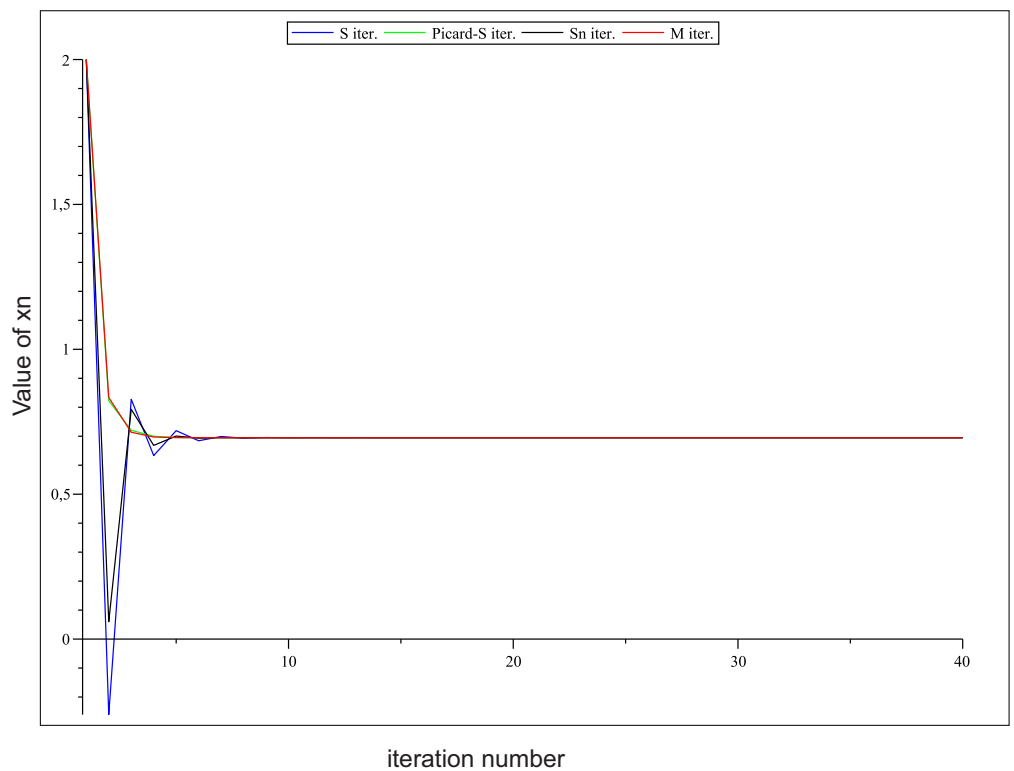

FIG. 3.1: Behaviour of the iterations given in Example 1

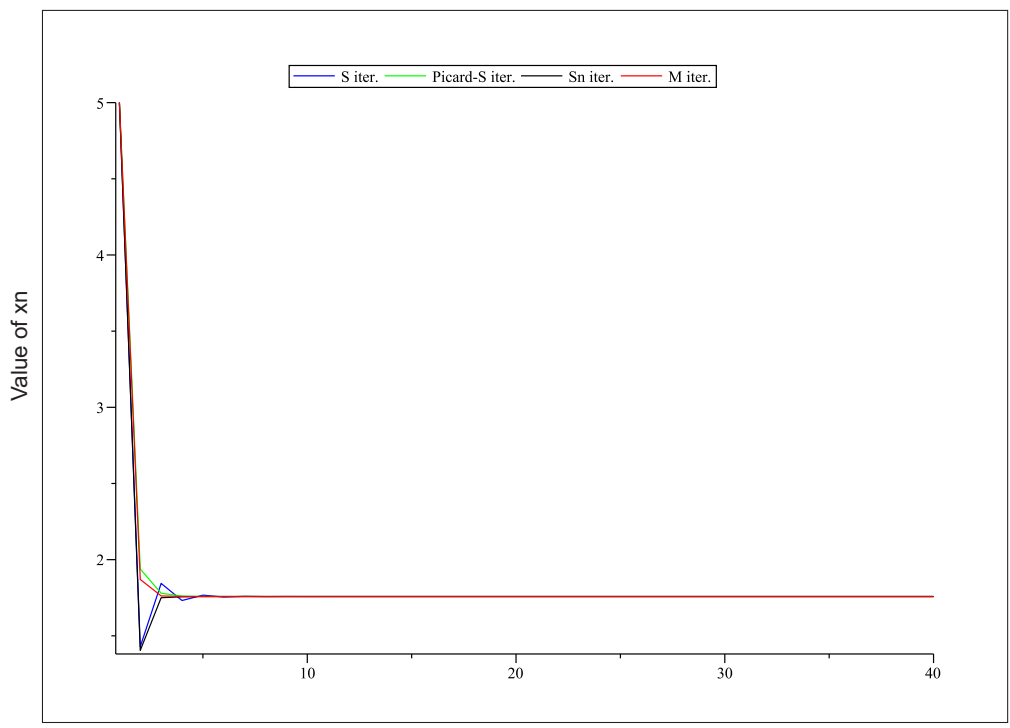

iteration number

FIG. 3.2: Behaviour of the iterations given in Example 2 


\section{R E F E R E N C E S}

1. M. ABBAS and T. NAZIR: A new faster iteration process applied to constrained minimization and feasibility problems. Mathematički Vesnik, 66 (2) (2014), 223234.

2. R. P. Agarwal, D. O'Regan and D. R. SAhu: Iterative construction of fixed points of nearly asymptotically nonexpansive mappings. J. Nonlinear Convex Anal. 8 (1) (2007), 61-79.

3. M. BASARIR and A. SAHIN: Some results of the new iterative scheme in hyperbolic space. Commun. Korean Math. Soc. 32 (4) (2017), 100-1024.

4. V. BERINDE: Picard iteration converges faster than Mann iteration for a class of quasi-contractive operators. Fixed Point Theory and Applications 2 (2001), 97-105.

5. R. Chugh, V. Kumar and S. Kumar: Strong Convergence of a new three step iterative scheme in Banach spaces. American Journal of Computational Mathematics 2 (2012), 345-357.

6. F. Gursoy and V. Karakaya: A Picard-S hybrid type iteration method for solving a differential equation with retarded argument (2014), arXiv:1403.2546v2

7. S. Ishikawa: Fixed points by a new iteration method. Proc. Am. Math. Soc. 44 (1974), 147-150.

8. I. KARAHAN and M. OZDEMIR. A general iterative method for approximation of fixed points and their applications Advances in Fixed Point Theory 3(3) (2013), 510-526.

9. W. R. Mann: Mean value methods in iteration. Proc. Am. Math. Soc. 4 (1953), 506-510.

10. M. A. NooR: New approximation schemes for general variational inequalities Journal of Mathematical Analysis and Applications 251 (2000), 217-229.

11. W. Phuengrattana and S. Suantai. On the rate of convergence of Mann, Ishikawa, Noor and SP-iterations for continuous functions on an arbitrary interval. Journal of Computational and Applied Mathematics 235 (2011), 3006-3014.

12. E. PICARD: Memoire sur la theorie des equations aux derivees partielles et la methodedes approximations successives. Journal de Mathematiques Pures et Appliquees. 6 (1890), 145-210.

13. D. R. SAHU and A. PETRUSEL: Strong convergence of iterative methods by strictly pseudo-contractive mappings in Banach spaces. Nonlinear Analysis: Theory, Methods Applications 74 (17) (2011), 6012-6023.

14. W. Sintunavarat and A. Pitea: On a new iteration scheme for numerical reckoning fixed points of Berinde mappings with convergence analysis. J. Nonlinear Sci. Appl. 9 (2016), 2553-2562.

15. S. Thianwan: Common fixed points of new iterations for two asymptotically nonexpansive nonself mappings in a Banach space. Journal of Computational and Applied Mathematics 224 (2009), 688-695.

16. K. Ullah Rm and M. Arshad: Numarical Reckoning Fixed Points for Suzuki's Generalized Nonexpansive Mappings via Iteration Process. Filomat, 32:1 (2018), 187-196. 
17. T. Zamfirescu: Fixed point theorems in metric spaces. Arch. Math. 23 (1972), 292-298.

Osman Alagoz

Bilecik Seyh Edebali University

Faculty of Science

Department of Mathematics

11000 Bilecik, Turkiye

osman.alagoz@bilecik.edu.tr

Birol Gunduz

Erzincan University

Faculty of Science

Department of Mathematics

24000 Erzincan, Turkiye

birolgndz@gmail.com

Sezgin Akbulut

Ataturk University

Faculty of Science

Department of Mathematics

25000 Erzurum, Turkiye

sezginakbulut@atauni.edu.tr 